\title{
Efficient Identity Based Ring Signature *
}

\author{
Sherman S.M. Chow ${ }^{\star \star}$, S.M. Yiu, and Lucas C.K. Hui \\ Department of Computer Science \\ The University of Hong Kong \\ Pokfulam, Hong Kong \\ \{smchow, smyiu, hui\}@cs.hku.hk
}

\begin{abstract}
Identity-based (ID-based) cryptosystems eliminate the need for validity checking of the certificates and the need for registering for a certificate before getting the public key. These two features are desirable especially for the efficiency and the real spontaneity of ring signature, where a user can anonymously sign a message on behalf of a group of spontaneously conscripted users including the actual signer.

In this paper, we propose a novel construction of ID-based ring signature which only needs two pairing computations for any group size. The proposed scheme is proven to be existential unforgeable against adaptive chosen message-and-identity attack under the random oracle model, using the forking lemma for generic ring signature schemes. We also consider its extension to support the general access structure.
\end{abstract}

Key words: Identity-based signature, ring signature, bilinear pairings, efficiency, real spontaneity, general access structure, anonymity

\section{Introduction}

Ring signature is a group-oriented signature with privacy concerns: a user can anonymously signs a message on behalf of a group of spontaneously conscripted users including the actual signer. Any verifier can be convinced that the message has been signed by one of the members in this group, but the actual signer remains unknown. However, the theory of ring signature faces two problems when it comes to reality.

In traditional public key infrastructure (PKI), the public key is usually a "random" string that is unrelated to the identity of the user, so there is a need for a trusted-by-all certificate authority (CA) to assure the

\footnotetext{
* This research is supported in part by the Areas of Excellence Scheme established under the University Grants Committee of the Hong Kong Special Administrative Region (HKSAR), China (Project No. AoE/E-01/99), two grants from the Research Grants Council of the HKSAR, China (Project No. HKU/7144/03E and $\mathrm{HKU} / 7136 / 04 \mathrm{E})$, and two grants from the Innovation and Technology Commission of the HKSAR, China (Project No. ITS/170/01 and UIM/145).

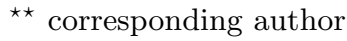


relationship between the cryptographic keys and the user. As a result, any verifier of a signature must obtain a copy of the user's certificate and check the validity of the certificate before checking the validity of the signature. In ring signature, not only the verifier must verify all the public keys of the group. The signer must do so as well or his/her anonymity is jeopardized (consider the extreme case that all certificates used are indeed invalid except the signer's one). The communication and the validation of a large number of public keys greatly affect the efficiency of the scheme. Moreover, real spontaneity is not always possible for ring signature under traditional PKI. The signer cannot spontaneously conscript users who have not registered for a certificate.

Identity-based (ID-based) ring signature solved these problems: the public key of each user is easily computable from a string corresponding to this user's identity (for example, an email address). This property avoids the necessity of certificates, and associates an implicit public key to each person over the world.

Unfortunately, the theory of ID-based ring signature still faces some obstacles in real application: ID-based ring signature schemes are usually derived from bilinear pairings, a powerful but computationally expensive primitive. The important properties of bilinear pairings and associated intractable problems are recalled in Section 3.

From the review in the next section, we know that the number of pairing computations of all existing ID-based ring signature from bilinear pairings grows linearly with the group size, which makes the efficiency of ID-based schemes over traditional schemes questionable. It is fair to say devising an ID-based ring signature using sublinear numbers of pairing computation remains an open question.

We close this open problem in this paper. An efficient ID-based ring signature is proposed in Section 5, which only takes two pairing operations for any group size, and the generation of the signature involves no pairing computations at all. The proposed scheme is proven to be existential unforgeable against adaptive chosen message-and-identity attack under the random oracle model. The framework and the security notion of IDbased ring signature are discussed in Section 4.

In the literature, 1 -out-of- $n$-groups ring signature was also considered, which supports an ad-hoc access structure consisting of groups of different sizes. The verifier can be convinced that the signature is generated from all members of a certain group, but cannot know which group has indeed participated in the signing. We notice that an ID-based ring signature for the general access structure can be implemented by an 1-out-of- $n$-groups 
ring signature. Extension of the proposed scheme to support this general access structure is shown in Section 6 .

\section{Related Work}

The first work on ID-based ring signature is in [13]. After that, [8] gave a more efficient construction, while [1] pointed out and fixed some small inconsistencies in [13] and [8]. Another ID-based ring signature scheme was proposed in [6]. An ID-based ring signature scheme for anonymous subsets (i.e. 1 -out-of- $n$-groups instead of 1 -out-of- $n$-individuals) was also considered in this work. The pairing operations in [6] can be executed in parallel, which is not possible in schemes like $[1,8,13]$.

Threshold ring signature is the $t$-out-of- $n$ threshold version of ring signature, where $t$ or more entities can jointly generate a valid signature but $t-1$ or fewer entities cannot. These schemes are applied in pervasive computing applications and mobile ad-hoc networks, where ad-hoc groups are very common. The first ID-based threshold ring signature scheme was proposed in [4]. It is robust and hence anyone can check whether the partial signature is valid for the construction of the final signature. Moreover, it supports trusted authority (TA) compatibility, which enables the signer to conscript non-participating signers under different TAs. The scheme's time and space complexity are up to the state-of-the-art of existing pairing-based ring signature and threshold ring signature, if not better. Actually, it was the most efficient (in terms of number of pairing operations required) ID-based ring signature scheme (when $t=1$ ).

Taken into account the total computational costs of the signature generation and verification, existing solutions $[1,4,6,8,13]$ need a number of pairing computations ranging from $n+1$ to $4 n-1$ where $n$ is the group size of the ring signature. Since pairing computation is usually the most expensive one among other computations in ID-based cryptosystems, this linear dependence with the group size is undesirable. We remark that this linear dependence also appears in non-ID-based ring signature schemes from bilinear pairings, for examples, $[2,9,12,14]$.

The efficiency gain in ring signature schemes is also beneficial to cryptographic schemes that are built on top of ring signature. Examples include multi-designated verifiers signature [7], non-interactive deniable ring authentication [10] and perfect concurrent signature [11].

In [5], an separable and anonymous ID-based key issuing protocol was proposed. The anonymity property assures that any eavesdropper cannot learn what is the identity associated with the private key being issued 
even though the key is not transmitted via a secure channel, which is an essential feature for ID-based ring signature. If the protocol reveals information about who has requested for his/her private key and who has not, the real spontaneity will be affected, as the actual signer cannot choose arbitrary any non-participating signer as other may know well that no one except the TA knows the corresponding private key.

\section{Preliminaries}

\subsection{Bilinear Pairings and Related Complexity Assumptions}

Bilinear pairing is an important primitive for many cryptographic schemes [1-14]. Here, we describe some of its key properties.

Let $\left(\mathbb{G}_{1},+\right)$ and $\left(\mathbb{G}_{2}, \cdot\right)$ be two cyclic groups of prime order $q$. The bilinear pairing is given as $\hat{e}: \mathbb{G}_{1} \times \mathbb{G}_{1} \rightarrow \mathbb{G}_{2}$, which satisfies the following properties:

1. Bilinearity: For all $P, Q, R \in \mathbb{G}_{1}, \hat{e}(P+Q, R)=\hat{e}(P, R) \hat{e}(Q, R)$, and $\hat{e}(P, Q+R)=\hat{e}(P, Q) \hat{e}(P, R)$.

2. Non-degeneracy: There exists $P, Q \in \mathbb{G}_{1}$ such that $\hat{e}(P, Q) \neq 1$.

3. Computability: It is efficient to compute $\hat{e}(P, Q) \forall P, Q \in \mathbb{G}_{1}$.

Definition 1. Given a generator $P$ of a group $\mathbb{G}$ and a 3-tuple $(a P, b P, c P)$, the Decisional Diffie-Hellman problem (DDHP) is to decide if $c=a b$.

Definition 2. Given a generator $P$ of a group $\mathbb{G}$ and a 2-tuple $(a P, b P)$, the Computational Diffie-Hellman problem (CDHP) is to compute abP.

Definition 3. We define $\mathbb{G}$ as a Gap Diffie-Hellman (GDH) group if $\mathbb{G}$ is a group such that DDHP can be solved in polynomial time but no algorithm can solve CDHP with non-negligible advantage within polynomial time.

We assume the existence of a bilinear map $\hat{e}: \mathbb{G}_{1} \times \mathbb{G}_{1} \rightarrow \mathbb{G}_{2}$ that one can solve Decisional Diffie-Hellman Problem in polynomial time.

\subsection{Forking Lemma for Ring Signature Schemes}

The unforgeability of (ID-based) ring signature schemes can be proven with the help of the forking lemma for generic ring signature scheme [6]. Here we review the required conditions for a ring signature scheme to be considered as generic. Denote $H(\cdot)$ be a cryptographic hash function that outputs $k$ bits, where $k$ is the security parameter. Consider a group $L$ 
of $n$ members $\left(L=\left\{I D_{1}, I D_{2}, \cdots, I D_{n}\right\}\right)$ and a message $m$, a generic ring signature scheme will produce ring signatures in the form of $\{L, m$, $\left.R_{1}, R_{2}, \cdots, R_{n}, h_{1}, h_{2}, \cdots, h_{n}, \sigma\right\}$ where for $i \in\{1,2, \cdots, n\}, R_{i}$ s are distinct and no $R_{i}$ can appear in a signature with probability greater than $2 / 2^{k} ; h_{i}=H\left(L, m, R_{i}\right)$ and $\sigma$ is dependent on all of $\bigcup\left\{R_{i}\right\}, \bigcup\left\{h_{i}\right\}$ and $m$.

Theorem 1 Consider a generic ring signature scheme using security parameter $k$. Let $\mathcal{A}$ be a probabilistic polynomial time algorithm which takes as the identity of each members in the group of $L$ and the public parameters that can ask for at most $Q$ queries to the random oracle; if $\mathcal{A}$ can produce a valid ring signature $\left\{L, m, R_{1}, \cdots, R_{n}, h_{1}, \cdots, h_{n}, \sigma\right\}$, for some $L^{*} \subset L$ of $n$ users within time bound $T$ and with non-negligible probability of success $\epsilon \geq \frac{7 C_{n}^{Q}}{2^{k}}$, where $C_{n}^{Q}$ is defined as the number of $n$ permutations of $Q$ elements, i.e., $C_{n}^{Q}=Q \times(Q-1) \times \cdots \times(Q-n+1)$. Then, within a time period of $2 T$ and with probability greater than $\frac{\epsilon^{2}}{66 C_{n}^{Q}}$, we can use $\mathcal{A}$ to obtain two valid ring signatures $\left\{L, m, R_{1}, \cdots, R_{n}, h_{1}, \cdots, h_{n}, \sigma\right\}$ and $\left\{L, m, R_{1}, \cdots, R_{n}, h^{\prime}{ }_{1}, \cdots, h_{n}^{\prime}, \sigma^{\prime}\right\}$ such that $h_{j} \neq h^{\prime}{ }_{j}$, for some $j \in\{1, \cdots, n\}$ and $h_{i}=h^{\prime}{ }_{i}$ for all $i \in\{1, \cdots, n\} \backslash\{j\}$.

In the practical implementation, we usually omit $\bigcup\left\{h_{i}\right\}$ in the ring signature as they can be correctly recovered during the verification process.

\section{Framework of ID-based Ring Signature Schemes}

\subsection{ID-based Ring Signature}

Framework An ID-based ring signature scheme consists of the following four algorithms: Setup, KeyGen, Sign, and Verify.

- Setup: On an unary string input $1^{k}$ where $k$ is a security parameter, it produces the master secret key $s$ and the common public parameters params, which include a description of a finite signature space and a description of a finite message space.

- KeyGen: On an input of the signer's identity $I D \in\{0,1\}^{*}$ and the master secret key $s$, it outputs the signer's secret signing key $S_{I D}$. (The corresponding public verification key $Q_{I D}$ can be computed easily by everyone.)

- Sign: On input of a message $m$, a group of $n$ users' identities $\bigcup\left\{I D_{i}\right\}$, where $1 \leq i \leq n$, and the secret keys of one members $S_{I D_{s}}$, where $1 \leq s \leq n$; it outputs an ID-based ring signature $\sigma$ on the message $m$. 
- Verify: On a ring signature $\sigma$, a message $m$ and the group of signers' identities $\bigcup\left\{I D_{i}\right\}$ as the input, it outputs $\top$ for "true" or $\perp$ for "false", depending on whether $\sigma$ is a valid signature signed by a certain member in the group $\bigcup\left\{I D_{i}\right\}$ on a message $m$.

These algorithms must satisfy the standard consistency constraint of IDbased ring signature scheme, i.e. if $\sigma=\operatorname{Sign}\left(m, \bigcup\left\{I D_{i}\right\}, S_{I D_{s}}\right)$, and $I D_{s} \in$ $\bigcup\left\{I D_{i}\right\}$, we must have $\operatorname{Verify}\left(\sigma, \bigcup\left\{I D_{i}\right\}, m\right)=\top$.

A secure ID-based ring signature scheme should be unforgeability and signer-ambiguous.

Security Notions The EUF-IDRS-CMIA2 game below formally defines the existential unforgeability of ID-based ring signature under adaptive chosen-message-and-identity attack.

\section{EUF-IDRS-CMIA2 Game:}

Setup: The challenger $\mathcal{C}$ takes a security parameter $k$ and runs the Setup to generate common public parameters params and also the master secret key s. $\mathcal{C}$ sends params to $\mathcal{A}$.

Attack: The adversary $\mathcal{A}$ can perform a polynomially bounded number of queries described below in an adaptive manner (that is, each query may depend on the responses to the previous queries).

- Hash functions queries: $\mathcal{A}$ can ask for the values of the hash functions (e.g. $H(\cdot)$ and $H_{0}(\cdot)$ in our proposed scheme) for any input.

- KeyGen: $\mathcal{A}$ chooses an identity ID. $\mathcal{C}$ computes KeyGen $(I D)=S_{I D}$ and sends the result to $\mathcal{A}$.

- Sign: $\mathcal{A}$ chooses a group of $n$ users' identities $\bigcup\left\{I D_{i}\right\}$ where $1 \leq i \leq n$, and any message $m . \mathcal{C}$ outputs an ID-based ring signature $\sigma$.

Forgery: The adversary $\mathcal{A}$ outputs an ID-based ring signature $\sigma$ and a group of $n$ users' identities $\bigcup\left\{I D_{i}\right\}$ where $1 \leq i \leq n$. The only restriction is that $\left(m, \bigcup\left\{I D_{i}\right\}\right)$ does not appear in the set of previous Sign queries and each of the secret keys in $\bigcup\left\{S_{I D_{i}}\right\}$ is never returned by any KeyGen query. i.e. no private keys in $\bigcup\left\{S_{I D_{i}}\right\}$ is known. It wins the game if $\operatorname{Verify}\left(\sigma, \bigcup\left\{I D_{i}\right\}\right)$ is equal to $\top$. The advantage of $\mathcal{A}$ is defined as the probability that it wins.

Definition 4. An ID-based ring signature scheme is said to satisfy the property of existential unforgeability against adaptive chosen-messageand-identity attacks (EUF-IDRS-CMIA2 secure) if no adversary has a non-negligible advantage in the EUF-IDRS-CMIA2 game. 
Definition 5. An ID-based ring signature scheme is said to have the unconditional signer ambiguity if for any group of $n$ users' identities $\bigcup\left\{I D_{i}\right\}$ where $1 \leq i \leq n$, any message $m$ and any signature $\sigma$, where $\sigma=\operatorname{Sign}\left(m, \bigcup\left\{I D_{i}\right\}\right)$; any verifier $\mathcal{A}$ even with unbounded computing resources, cannot identify the actual signer with probability better than a random guess. That is, $\mathcal{A}$ can only output the actual signer indexed by $s$ with probability no better than $\frac{1}{n}\left(\frac{1}{n-1}\right.$ is $\mathcal{A}$ is in the signers group.

\subsection{ID-Based Ring Signature for General Access Structure}

Framework An ID-based ring signature scheme for the general access structure consists of the following four algorithms: Setup, KeyGen, Sign, and Verify.

- Setup: Same as Setup of ID-based ring signature scheme.

- KeyGen: Same as KeyGen of ID-based ring signature scheme.

- Sign: On input of a message $m, n$ groups of users' identities $\bigcup\left\{\mathcal{U}_{i}\right\}$, where $\mathcal{U}_{i}=\bigcup\left\{I D_{i_{j}}\right\}$ for $1 \leq i \leq n$, and the secret keys $\bigcup\left\{S_{I D_{s_{j}}}\right\}$ of each signer in one of the groups $\mathcal{U}_{s}$, where $1 \leq s \leq n$; it outputs an ID-based ring signature for access structure $\bigcup\left\{\mathcal{U}_{i}\right\}$ on the message $m$.

- Verify: On input of a ring signature $\sigma$, a message $m$ and $n$ groups of users' identities $\bigcup\left\{\mathcal{U}_{i}\right\}$, where $\mathcal{U}_{i}=\bigcup\left\{I D_{i_{j}}\right\}$ for $1 \leq i \leq n$, it outputs $\top$ for "true" or $\perp$ for "false", depending on whether $\sigma$ is a valid signature signed by all members of a certain group in $\bigcup\left\{\mathcal{U}_{i}\right\}$ on a message $m$.

These algorithms must satisfy the standard consistency constraint of IDbased ring signature scheme for the general access structure, i.e. if $\sigma=$ $\operatorname{Sign}\left(m, \bigcup\left\{\mathcal{U}_{i}\right\}, \bigcup\left\{S_{I D_{s_{j}}}\right\}\right)$ and $\bigcup\left\{I D_{s_{j}}\right\} \in \bigcup\left\{\mathcal{U}_{i}\right\}$ we must get "true" from the verification algorithm taking the signature, the message and the groups of identities as the input, i.e. $\operatorname{Verify}\left(\sigma, \bigcup\left\{\mathcal{U}_{i}\right\}, m\right)=\top$.

We say an ID-based ring signature scheme for the general access structure is secure if it satisfies unforgeability and signer ambiguity.

Security Notions The following EUF-IDRSG-CMIA2 game formally defines the existential unforgeability of ID-based ring signature under adaptive chosen-message-and-identity attack.

\section{EUF-IDRSG-CMIA2 Game:}

Setup: The challenger $\mathcal{C}$ takes a security parameter $k$ and runs the Setup to generate common public parameters params and also the master secret key s. $\mathcal{C}$ sends params to $\mathcal{A}$. 
Attack: The adversary $\mathcal{A}$ can perform a polynomially bounded number of queries described below in an adaptive manner (that is, each query may depend on the responses to the previous queries).

- Hash functions queries: $\mathcal{A}$ can ask for the values of the hash functions (e.g. $H(\cdot)$ and $H_{0}(\cdot)$ in our proposed scheme) for any input.

- KeyGen: $\mathcal{A}$ chooses an identity ID. $\mathcal{C}$ computes KeyGen $(I D)=S_{I D}$ and sends the result to $\mathcal{A}$.

- Sign: $\mathcal{A}$ chooses $n$ groups of users' identities $\bigcup\left\{\mathcal{U}_{i}\right\}$, where $\mathcal{U}_{i}=$ $\bigcup\left\{I D_{i_{j}}\right\}$ for $1 \leq i \leq n$, and any message $m$. $\mathcal{C}$ outputs an ID-based ring signature for the general access structure $\sigma$.

Forgery: The adversary $\mathcal{A}$ outputs an ID-based ring signature $\sigma$ and $n$ groups of users' identities $\bigcup\left\{\mathcal{U}_{i}\right\}$, where $\mathcal{U}_{i}=\bigcup\left\{I D_{i_{j}}\right\}$ for $1 \leq i \leq n$. The only restriction is that $\left(m, \bigcup\left\{\mathcal{U}_{i}\right\}\right)$ does not appear in the set of previous Sign queries and for each group of identities $\bigcup\left\{\mathcal{U}_{i}\right\}$, at least one secret key in $\bigcup\left\{S_{I D_{i_{j}}}\right\}$ is never returned by any KeyGen query. It wins the game if $\operatorname{Verify}\left(\sigma, \bigcup\left\{\mathcal{U}_{i}\right\}\right)$ is equal to $T$. The advantage of $\mathcal{A}$ is defined as the probability that it wins.

Definition 6. An ID-based ring signature scheme for the general access structure is existentially unforgeable against adaptive chosen-messageand-identity attacks (EUF-IDRSG-CMIA2 secure) if no adversary has a non-negligible advantage in the EUF-IDRSG-CMIA2 game.

Definition 7. An ID-based ring signature scheme for the general access structure is said to have the unconditional group of signers ambiguity if for any $n$ groups of users' identities $\bigcup\left\{\mathcal{U}_{i}\right\}$, where $\mathcal{U}_{i}=\bigcup\left\{I D_{i_{j}}\right\}$ for $1 \leq$ $i \leq n$, any message $m$ and any signature $\sigma$, where $\sigma=\operatorname{Sign}\left(m, \bigcup\left\{\mathcal{U}_{i}\right\}\right)$;

any verifier $\mathcal{A}$ not from the actual signer group, even with unbounded computing resources, cannot identify the actual group of signers with probability better than a random guess. That is, $\mathcal{A}$ can only output the actual signers group indexed by $s$ with probability no better than $\frac{1}{n}$.

\section{Efficient ID-based Ring Signature}

\subsection{Construction}

Define $\mathbb{G}_{1}, \mathbb{G}_{2}$, and $\hat{e}(\cdot, \cdot)$ as in the Section 3 where $\mathbb{G}_{1}$ is a GDH group. $H(\cdot)$ and $H_{0}(\cdot)$ are two cryptographic hash functions where $H:\{0,1\}^{*} \rightarrow$ $\mathbb{G}_{1}$ and $H_{0}:\{0,1\}^{*} \rightarrow \mathbb{Z}_{q}^{*}$. 
Setup: The TA randomly chooses $x \in_{R} \mathbb{Z}_{q}^{*}$, keeps it as the master secret key and computes the corresponding public key $P_{p u b}=x P$. The system parameters are: $\left\{\mathbb{G}_{1}, \mathbb{G}_{2}, \hat{e}(\cdot, \cdot), q, P, P_{\text {pub }}, H(\cdot), H_{0}(\cdot)\right\}$.

KeyGen: The signer with identity $I D \in\{0,1\}^{*}$ submits $I D$ to TA. TA sets the signer's public key $Q_{I D}$ to be $H(I D) \in \mathbb{G}_{1}$, computes the signer's private signing key $S_{I D}$ by $S_{I D}=x Q_{I D}$. Then TA sends the private signing key to the signer via a secure channel, or using the secure and anonymous protocol proposed in [5].

Sign: Let $L=\left\{I D_{1}, I D_{2}, \cdots, I D_{n}\right\}$ be the set of all identities of $n$ users. The actual signer, indexed by $s$ (i.e. his/her public key is $Q_{I D_{s}}=$ $H\left(I D_{s}\right)$ ), carries out the following steps to give an ID-based ring signature on behalf of the group $L$.

1. Choose $U_{i} \in_{R} \mathbb{G}_{1}$, compute $h_{i}=H_{0}\left(m\|L\| U_{i}\right) \forall i \in\{1,2, \cdots, n\} \backslash\{s\}$.

2. Choose $r_{s}^{\prime} \in_{R} \mathbb{Z}_{q}^{*}$, compute $U_{s}=r_{s}^{\prime} Q_{I D_{s}}-\sum_{i \neq s}\left\{U_{i}+h_{i} Q_{I D_{i}}\right\}$.

3. Compute $h_{s}=H_{0}\left(m\|L\| U_{s}\right)$ and $V=\left(h_{s}+r_{s}^{\prime}\right) S_{I D_{s}}$.

4. Output the signature on $m$ as $\sigma=\left\{\bigcup_{i=1}^{n}\left\{U_{i}\right\}, V\right\}$.

Verify: A verifier can check the validity of a signature $\sigma=\left\{\bigcup_{i=1}^{n}\left\{U_{i}\right\}, V\right\}$ for the message $m$ and a set of identities $L$ as follows.

1. Compute $h_{i}=H_{0}\left(m|| L|| U_{i}\right) \forall i \in\{1,2, \cdots, n\}$.

2. Checking whether $\hat{e}\left(P_{p u b}, \sum_{i=1}^{n}\left(U_{i}+h_{i} Q_{I D_{i}}\right)\right)=\hat{e}(P, V)$.

3. Accept the signature if it is true, reject otherwise.

\subsection{Efficiency}

We consider the costly operations which include point addition on $\mathbb{G}_{1}\left(\mathbb{G}_{1}\right.$ Add), point scalar multiplication on $\mathbb{G}_{1}\left(\mathbb{G}_{1}\right.$ Mul $)$, multiplication on $\mathbb{G}_{2}$ or $\mathbb{Z}_{q}\left(\mathbb{G}_{2} / \mathbb{Z}_{q} \mathrm{Mul}\right)$, hashing into the group (Hash) and pairing operation (Pairing). We use the MapToPoint hash operation in BLS short signature scheme [3]. Before our proposal, the scheme that requires the least number of pairing operations is [4]. Table 1 shows a summary of the efficiency of our proposed scheme. Taken into account the total cost of the signature generation and verification, we can see that our proposed scheme is the only scheme using a constant number of pairing operations, and with the least total amount of other operations. Moreover, our scheme supports parallel operations for the computation about non-participating signers' parts like [4] and [6], which is not possible in schemes like $[1,8,13]$. 


\begin{tabular}{|l|c|c|c|c|c|c|c|}
\hline Schemes & $G_{1}$ Add & $G_{1}$ Mul & $G_{2} / Z_{q}$ Mul & Hash & Pairing & Parallelism & Proof \\
\hline \hline Zhang-Kim [13] & 1 & $2 n$ & $2 n-1$ & $2 n$ & $4 n-1$ & $\times$ & $\checkmark$ \\
\hline \hline Lin-Wu [8] & $2 n-1$ & $2 n$ & $3 n$ & 0 & $2 n+1$ & $\times$ & $\times$ \\
\hline \hline Herranz-Sáez [6] & $3 n-1$ & $2 n$ & $n$ & 0 & $n+3$ & $\checkmark$ & $\checkmark$ \\
\hline \hline Awasthi-Lai [1] & $2 n-1$ & $2 n+1$ & $2 n-1$ & 0 & $4 n-1$ & $\times$ & $\times$ \\
\hline \hline Chow et al. $[4](t=1)$ & $2 n$ & $4 n$ & $n-1$ & 0 & $n+1$ & $\checkmark$ & $\checkmark$ \\
\hline \hline Proposed Scheme & $4 n-3$ & $2 n+1$ & 0 & 0 & 2 & $\checkmark$ & $\checkmark$ \\
\hline
\end{tabular}

Table 1. Comparison of ID-based Ring Signature from Bilinear Pairings

Considering the signature size, we share the same order of space complexities as all other schemes we considered $[1,4,6,8,13]$, we are not sacrificing the signature size for lowering time complexity. A final remark for the comparison is that all the schemes with formally proven security employ the forking technique like [6] in their proofs.

\subsection{Existential Unforgeability and Signer Ambiguity}

We summarize our proposed scheme's security in the following theorems.

Theorem 2 In the random oracle model (the hash functions are modeled as random oracles), if there is an algorithm $\mathcal{A}$ that can win the EUFIDRS-CMIA2 game with non-negligible probability by making a valid ring signature of group size $n^{\prime}$, in polynomial time with probability $\epsilon_{\mathcal{A}}$, asking at most $q_{S}$ sign queries, $q_{H} H_{1}$ queries (including those implicitly asked by sign queries), $q_{E}$ key generation queries and $q_{I}$ identity hashing queries, CDHP can be solved with non-negligible probability in polynomial time.

Theorem 3 Our ID-based ring signature scheme has the unconditional signer ambiguity property.

\section{Extension}

Now we show the extension to support an ad-hoc access structure consists of groups of different sizes. We employ the idea from [6], where the access structure $\mathcal{U}$ is defined as $\left\{\mathcal{U}_{1}, \mathcal{U}_{2}, \cdots \mathcal{U}_{d}\right\}$ (where $\mathcal{U}_{i}$ denotes a set of signers) and all the members of a particular set in $\mathcal{U}$ (says $\mathcal{U}_{s}$, where $1 \leq s \leq d$ ) participate in the signing. The signature can convince any one that all the members of a certain group in $\mathcal{U}$ have cooperated to give the signature, but does not know which group is signing. 


\subsection{Construction}

The Setup and Keygen algorithm are the same as the basic scheme, except the security parameter in Setup should be chosen with the maximum number of subsets supported $(n)$ in mind. Below are the descriptions of Sign and Verify algorithm.

Sign: Let $\mathcal{U}_{s}=\left\{I D_{1}, I D_{2}, \cdots, I D_{n_{s}}\right\}$ be the set of all identities of $n_{s}$ users. They choose an access structure $\mathcal{U}$ is defined as $\left\{\mathcal{U}_{1}, \mathcal{U}_{2}, \cdots \mathcal{U}_{d}\right\}$ where $\mathcal{U}_{s} \in \mathcal{U}$. The ID-based ring signature for the access structure $\mathcal{U}$ can be generated as follows.

1. Compute $Y_{i}=\sum_{I D_{j} \in \mathcal{U}_{i}}\left(Q_{I D_{j}}\right), \forall i \in\{1,2, \cdots, d\}$.

2. Choose $U_{i} \in_{R} \mathbb{G}_{1}$, compute $h_{i}=H_{0}\left(m|| \mathcal{U} \| U_{i}\right) \forall i \in\{1,2, \cdots, d\} \backslash\{s\}$.

3. Each signer $I D_{s_{k}} \in \mathcal{U}_{s}$ chooses $r_{s_{k}}^{\prime} \in R \mathbb{Z}_{q}^{*}$ and computes $U_{s_{k}}=$ $r_{s_{k}}^{\prime} Q_{I D_{s_{k}}}, \forall k \in\left\{1,2, \cdots, n_{s}\right\}$.

4. Any particular signer who got the knowledge of $\bigcup_{s_{k}=1}^{n_{s}}\left\{U_{s_{k}}\right\}$ computes $U_{s}=\sum_{s_{k}=1}^{n_{s}}\left(U_{s_{k}}\right)-\sum_{i \neq s}\left\{U_{i}+h_{i} Y_{i}\right\}$ and $h_{s}=H_{0}\left(m\|\mathcal{U}\| U_{s}\right)$.

5. Each signer $I D_{s_{k}} \in \mathcal{U}_{s}$ computes $V_{s_{k}}=\left(h_{s}+r_{s_{k}}^{\prime}\right) S_{I D_{s_{k}}}$.

6. Output the signature on $m$ as $\sigma=\left\{\bigcup_{i=1}^{d}\left\{U_{i}\right\}, V=\sum_{I D_{s_{k}} \in \mathcal{U}_{s}}\left(V_{s_{k}}\right)\right\}$.

Verify: A verifier can check the validity of a signature $\sigma=\left\{\bigcup_{i=1}^{d}\left\{U_{i}\right\}, V\right\}$ for the message $m$ and the access structure $\mathcal{U}$ as follows.

1. Compute $h_{i}=H_{0}\left(m|| \mathcal{U} \| U_{i}\right) \forall i \in\{1,2, \cdots, d\}$.

2. Checking whether $\hat{e}\left\{P_{p u b}, \sum_{i=1}^{d}\left[U_{i}+h_{i} \sum_{I D_{j} \in \mathcal{U}_{i}}\left(Q_{I D_{j}}\right)\right]\right\}=\hat{e}(P, V)$.

3. Accept the signature if it is true, reject otherwise.

\subsection{Robustness}

Robustness is often desirable in multi-party cryptographic protocols. If the scheme is not robust, the misbehavior of any participating signer cannot be detected, and the final signature will be invalid even there is only one misbehaving signer. In our scheme, the partial signature $\sigma_{j}=\left\{h_{s}, U_{s_{k}}, V_{s_{k}}\right\}$ generated by the signer $I D_{s_{k}}$ can be verified easily by checking whether $\hat{e}\left(U_{s_{k}}+h_{j} Q_{I D_{s_{k}}}, P_{p u b}\right)=\hat{e}\left(P, V_{s_{k}}\right)$ holds.

\subsection{Security}

The scheme's signer ambiguity can be shown in a similar manner as the cases in our basic scheme. The proof of existential unforgeability is 
basically the same as that of our basic scheme. Due to page limit, we only highlight the differences here.

The first difference is concerned with the requirement on the forger's signature. For our basic scheme, the forger should not know all the private key associated with the signature, and this happens with probability (1$\zeta)^{n^{\prime}}$, where $n^{\prime}$ represents the total number of members associated with the forged signature. For our extended scheme, the forger must not know at least one private key for all group of signers, and the corresponding probability is $\left(1-\zeta^{n_{1}}\right)\left(1-\zeta^{n_{2}}\right) \cdots\left(1-\zeta^{n_{d}}\right)$ where $n_{i}{ }^{\prime}$ is the group size of the $i$-th group of users. Suppose $N^{\prime}=\sum_{i=1}^{d} n_{i}{ }^{\prime}$, this probability is greater than $(1-\zeta)^{n_{1}{ }^{\prime}}(1-\zeta)^{n_{2}{ }^{\prime}} \cdots(1-\zeta)^{n_{d}{ }^{\prime}}=(1-\zeta)^{N^{\prime}}$. Hence the $n^{\prime}$ parameter in the proof can be replaced by $N^{\prime}$, which represents the total number of members in all $d$ groups associated with the forged signature.

The second difference is about the solving of computational DiffieHellman problem. For our basic scheme, $a b P$ is computed by $y_{s}{ }^{-1}\left(h_{s}-\right.$ $\left.h_{s}^{\prime}\right)^{-1}\left(V-V^{\prime}\right)$. For our extended scheme, $\left(h_{s}-h_{s}^{\prime}\right)^{-1}\left(V-V^{\prime}\right)$ only gives the "private key" corresponding to $Y_{s}=\sum_{I D_{j} \in \mathcal{U}_{s}}\left(Q_{I D_{j}}\right)$. To obtain $a b P$, we should subtract other known private keys of this $s$-th group from this value. Suppose the unknown private key is indexed by $s_{k}$, we can compute $a b P$ by $y_{s_{k}}{ }^{-1}\left\{\left(h_{s}-h_{s}^{\prime}\right)^{-1}\left(V-V^{\prime}\right)-\sum_{I D_{j} \in \mathcal{U}_{s} \backslash\left\{I D_{s_{k}}\right\}}\left[\left(y_{j}\right)(b P)\right]\right\}$, where $y_{j}$ s can be found by looking up the list $L$.

\section{Conclusion}

For ring signature schemes to be practical, we need to eliminate the need for validity checking of the certificates and the need for registering for a certificate before getting the public key. ID-based solutions can provide these two features. Nonetheless, all of the existing proposals of ID-based ring signature are computationally inefficient, since the number of pairing computations grows linearly with the group size. This paper closes the open problem of devising an ID-based ring signature using sublinear numbers of pairing computation. We construct an efficient IDbased ring signature which only needs two pairing computations for any group size. The proposed scheme is proven to be existential unforgeable against adaptive chosen message-and-identity attack under the random oracle model, using the forking lemma for generic ring signature schemes. We also consider its extension to support the general access structure. Future research direction include further improving the efficiency in the generation or the verification of an ID-based ring signature. 


\section{References}

1. Amit K Awasthi and Sunder Lal. ID-based Ring Signature and Proxy Ring Signature Schemes from Bilinear Pairings. Cryptology ePrint Archive, Report 2004/184, 2004. Available at http://eprint.iacr.org.

2. Dan Boneh, Craig Gentry, Ben Lynn, and Hovav Shacham. Aggregate and Verifiably Encrypted Signatures from Bilinear Maps. In Eli Biham, editor, Advances in Cryptology - EUROCRYPT 2003, International Conference on the Theory and Applications of Cryptographic Techniques, Warsaw, Poland, May 48, 2003, Proceedings, volume 2656 of Lecture Notes in Computer Science, pages 416-432. Springer, 2003.

3. Dan Boneh, Ben Lynn, and Hovav Shacham. Short Signatures from the Weil Pairing. In Colin Boyd, editor, Advances in Cryptology - ASIACRYPT 2001, 7th International Conference on the Theory and Application of Cryptology and Information Security, Gold Coast, Australia, December 9-13, 2001, Proceedings, volume 2248 of Lecture Notes in Computer Science, pages 514-532. Springer, 2001.

4. Sherman S.M. Chow, Lucas C.K. Hui, and S.M. Yiu. Identity Based Threshold Ring Signature. In Choonsik Park and Seongtaek Chee, editors, Information Security and Cryptology - ICISC 2004, 7th International Conference Seoul, Korea, December 2-3, 2004, Revised Papers, volume 3506 of Lecture Notes in Computer Science, pages 218-232. Springer, 2004.

5. Ai fen Sui, Sherman S.M. Chow, Lucas C.K. Hui, S.M. Yiu, K.P. Chow, W.W. Tsang, C.F. Chong, K.H. Pun, and H.W. Chan. Separable and Anonymous Identity-Based Key Issuing. In 1st International Workshop on Security in Networks and Distributed Systems (SNDS 2005), in conjunction with 11th International Conference on Parallel and Distributed Systems (ICPADS 2005), July 20-22 2005, Fukuoka, Japan. IEEE Computer Society, 2005.

6. Javier Herranz and Germán Sáez. New Identity-Based Ring Signature Schemes. In Javier Lopez, Sihan Qing, and Eiji Okamoto, editors, Information and Communications Security, 6th International Conference, ICICS 2004, Malaga, Spain, October 27-29, 2004, Proceedings, volume 3269 of Lecture Notes in Computer Science, pages 27-39, Malaga, Spain, October 2004. Springer-Verlag. Preliminary version available at Cryptology ePrint Archive, Report 2003/261.

7. Fabien Laguillaumie and Damien Vergnaud. Multi-designated Verifiers Signatures. In Javier Lopez, Sihan Qing, and Eiji Okamoto, editors, Information and Communications Security, 6th International Conference, ICICS 2004, Malaga, Spain, October 27-29, 2004, Proceedings, volume 3269 of Lecture Notes in Computer Science, pages 495-507, Malaga, Spain, October 2004. Springer-Verlag.

8. Chih-Yin Lin and Tzong-Chen Wu. An Identity-based Ring Signature Scheme from Bilinear Pairings. Cryptology ePrint Archive, Report 2003/117, 2003. Available at http://eprint.iacr.org.

9. Joseph K. Liu and Duncan S. Wong. On the Security Models of (Threshold) Ring Signature Schemes. In Choonsik Park and Seongtaek Chee, editors, Information Security and Cryptology - ICISC 2004, 7th International Conference Seoul, Korea, December 2-3, 2004, Revised Papers, volume 3506 of Lecture Notes in Computer Science. Springer, 2004.

10. Willy Susilo and Yi Mu. Non-Interactive Deniable Ring Authentication. In Jong In Lim and Dong Hoon Lee, editors, Information Security and Cryptology - ICISC 2003, 6th International Conference Seoul, Korea, November 27-28, 2003, Revised Papers, volume 2971 of Lecture Notes in Computer Science, pages 386-401, Seoul, Korea, 2004. Springer-Verlag. 
11. Willy Susilo, Yi Mu, and Fangguo Zhang. Perfect Concurrent Signature Schemes. In Javier Lopez, Sihan Qing, and Eiji Okamoto, editors, Information and Communications Security, 6th International Conference, ICICS 2004, Malaga, Spain, October 27-29, 2004, Proceedings, volume 3269 of Lecture Notes in Computer Science, pages 14-26, Malaga, Spain, October 2004. Springer-Verlag.

12. Jing $\mathrm{Xu}$, Zhenfeng Zhang, and Dengguo Feng. A Ring Signature Scheme Using Bilinear Pairings. In Chae Hoon Lim and Moti Yung, editors, Information Security Applications, 5th International Workshop, WISA 2004, Revised Papers, volume 3325 of Lecture Notes in Computer Science, pages 163-172, Jeju Island, Korea, August 2004. Springer-Verlag.

13. Fangguo Zhang and Kwangjo Kim. ID-Based Blind Signature and Ring Signature from Pairings. In Yuliang Zheng, editor, Advances in Cryptology ASIACRYPT 2002, 8th International Conference on the Theory and Application of Cryptology and Information Security, Queenstown, New Zealand, December 15, 2002, Proceedings, volume 2501 of Lecture Notes in Computer Science, pages 533-547. Springer, 2002.

14. Fangguo Zhang, Reihaneh Safavi-Naini, and Willy Susilo. An Efficient Signature Scheme from Bilinear Pairings and Its Applications. In Feng Bao, Robert H. Deng, and Jianying Zhou, editors, Public Key Cryptography - PKC 2004, 7th International Workshop on Theory and Practice in Public Key Cryptography, Singapore, March 1-4, 2004, volume 2947 of Lecture Notes in Computer Science, pages 277-290. Springer, 2004.

\section{Appendix}

Proof of Theorem 2. Suppose the challenger $\mathcal{C}$ receives a random instance $(P, a P, b P)$ of the CDHP and has to compute the value of $a b P . \mathcal{C}$ will run $\mathcal{A}$ as a subroutine and act as $\mathcal{A}$ 's challenger in the EUF-IDRS-CMIA2 game. During the game, $\mathcal{A}$ will consult $\mathcal{C}$ for answers to the random oracles $H$ and $H_{0}$. Roughly speaking, these answers are randomly generated, but to maintain the consistency and to avoid collision, $\mathcal{C}$ keeps three lists to store the answers used. We assume $\mathcal{A}$ will ask for $H(I D)$ before $I D$ is used in any other queries.

$\mathcal{C}$ gives $\mathcal{A}$ the system parameters with $P_{p u b}=b P$. The value $b$ is unknown to $\mathcal{C}$, which simulates the master key value for the TA.

$H$ requests: We embed part of the challenge $a P$ in the answer of many $H$ queries. When $\mathcal{A}$ asks queries on the hash value of identity $I D, \mathcal{C}$ picks $y_{i} \in_{R} \mathbb{Z}_{q}^{*}$ and repeats the process until $y_{i}$ is not in the list $L_{1} \cdot \mathcal{C}$ then flips a coin $W \in\{0,1\}$ that yields 0 with probability $\zeta$ and 1 with probability $1-\zeta$. ( $\zeta$ will be determined later.) If $W=0$ then the hash value $H(I D)$ is defined as $y_{i} P$; else if $W=1$ then returns $H(I D)=y_{i}(a P)$. In either case, $\mathcal{C}$ stores $\left(I D, y_{i}, W\right)$ in the list $L$. 
Note that when $W=0$, the associated private key is $y_{i}(b P)$ which $\mathcal{C}$ knows how to compute. But when $W=1$, since both $a$ and $b$ are unknown to $\mathcal{C}$, a KeyGen request on this identity will make $\mathcal{C}$ fail.

$H_{0}$ requests: When $\mathcal{A}$ asks queries on these hash values, $\mathcal{C}$ checks the corresponding list $L_{2}$. If an entry for the query is found, the same answer will be given to $\mathcal{A}$; otherwise, a randomly generated value will be used as an answer to $\mathcal{A}$, the query and the answer will then be stored in the list.

Sign requests: $\mathcal{A}$ chooses a group of $n$ users' identities $L=\bigcup\left\{I D_{i}\right\}$ where $1 \leq i \leq n$, and any message $m$. On input of $(L, m), \mathcal{C}$ outputs an ID-based ring signature $\sigma$ as follows.

1. Choose an index $s \in_{R}\{1,2, \cdots, n\}$.

2. Choose $U_{i} \in_{R} \mathbb{G}_{1}$, compute $h_{i}=H_{0}\left(m\|L\| U_{i}\right) \forall i \in\{1,2, \cdots, n\} \backslash\{s\}$.

3. Choose $h_{s}^{\prime} \in_{R} \mathbb{Z}_{q}^{*}$ and $z \in_{R} \mathbb{Z}_{q}^{*}$, compute $U_{s}=z P-h_{s}^{\prime} Q_{I D_{s}}-$ $\sum_{i \neq s}\left\{U_{i}+h_{i} Q_{I D_{i}}\right\}$.

4. Store the relationship $h_{s}=H_{0}\left(m\|L\| U_{s}\right)$ to the list $L_{2}$ and compute $V=z(b P)$, repeat Step 3 in case collision occurs.

5. Output the signature on $m$ as $\sigma=\left\{\bigcup_{i=1}^{n}\left\{U_{i}\right\}, V\right\}$.

Finally, $\mathcal{A}$ outputs a forged signature $\sigma=\left\{\bigcup_{i=1}^{n}\left\{U_{i}\right\}, V\right\}$ that is signed by a certain member in the group $\bigcup\left\{I D_{i}\right\}$ where $Q_{I D_{i}}=H\left(I D_{i}\right)=$ $y_{i}(a P) \forall i \in\{1,2, \cdots, n\}$, i.e. $\mathcal{A}$ has not requested for any one of the private keys of members in the group.

Solving CDHP : It follows from the forking lemma for generic ring signature schemes [6] that if $\epsilon_{\mathcal{C}} \geq 7 C_{n^{\prime}}^{q_{H}} / 2^{k}$, and $\mathcal{A}$ can give a valid forged signature within time $T_{\mathcal{A}}$ in the above interaction, then we can construct another algorithm $\mathcal{A}^{\prime}$ that outputs within time $2 T_{\mathcal{A}}$ two signed messages $\sigma=$ $\left\{\bigcup_{i=1}^{n}\left\{U_{i}\right\}, V\right\}$ and $\sigma^{\prime}=\left\{\bigcup_{i=1}^{n}\left\{U_{i}\right\}, V^{\prime}\right\}$ and with at least $\epsilon_{\mathcal{C}}{ }^{2} / 66 C_{n^{\prime}}^{q_{H}}$ probability. Suppose $h_{i}=H_{0}\left(m\|L\| U_{i}\right)$ and $h_{i}^{\prime}=H_{0}\left(m\|L\| U_{i}\right)$ for all $i \in\{1,2, \cdots, n\}$, we have $h_{i}=h_{i}^{\prime}$ for all $i \in\{1,2, \cdots, n\} \backslash\{s\}$. Given $\mathcal{A}^{\prime}$ derived from $\mathcal{A}$, we can solve the CDHP by computing $a b P=y_{s}{ }^{-1}\left(h_{s}-\right.$ $\left.h_{s}^{\prime}\right)^{-1}\left(V-V^{\prime}\right)$, where $y_{s}$ can be found by looking for $I D_{s}$ in the list $L$.

Probability of success : Now we determine the value of $\zeta$. The probability that $\mathcal{C}$ does not fail in all the $q_{E}$ private key extraction queries is $\zeta^{q_{E}}$, and the probability that $\mathcal{A}$ forged a signature that $\mathcal{C}$ does not know all the corresponding private keys involved in the signature is $(1-\zeta)^{n^{\prime}}$. So the combined probability is $\zeta^{q_{E}}(1-\zeta)^{n^{\prime}}$. By simple differentiation, we find the value of $\zeta$ that maximize this probability is $\frac{q_{E}}{q_{E}+n^{\prime}}$ and the maximized probability is $\left(1-\frac{n^{\prime}}{q_{E}+n^{\prime}}\right)^{q_{E}+n^{\prime}}\left(\frac{n^{\prime}}{q_{E}}\right)^{n^{\prime}}$. 
The probability for $\mathcal{C}$ not to fail in all the $q_{S}$ sign queries is $(1-$ $\left.q_{H} \frac{2}{2^{k}}\right)^{q_{S}}$, which is greater than $\left(1-\frac{q_{S} q_{H}}{2^{k-1}}\right)$. For very large $q_{E}$, the probability for $\mathcal{C}$ to succeed is $\epsilon_{\mathcal{C}}=\epsilon_{\mathcal{A}}\left(\frac{n^{\prime}}{e q_{E}}\right)^{n^{\prime}}\left(1-\frac{q_{S} q_{H}}{2^{k-1}}\right)$.

Proof of Theorem 3. Since $\bigcup_{i \neq s}\left\{U_{i}\right\}$ and also $r^{\prime}{ }_{s}$ are randomly generated, hence $\bigcup_{i=1}^{n}\left\{U_{i}\right\}$ are also uniformly distributed.

It remains to consider whether $V=\left(h_{s}+r^{\prime}{ }_{s}\right) S_{I D_{s}}$ leaks information about the actual signer. We focus on the value of $V-h_{s} S_{I D_{s}}=r^{\prime}{ }_{s} S_{I D_{s}}$ as $h_{s}$ is publicly computable. Obviously, $r_{s}^{\prime} S_{I D_{s}}$ is related to $U_{s}$. Any one can compute the value of $r^{\prime}{ }_{s} Q_{I D_{s}}$ by $U_{s}+\sum_{i \neq s}\left(U_{i}+h_{i} Q_{I D_{i}}\right)$. Together with the fact that the bilinearity can relate $r_{s}^{\prime} S_{I D_{s}}$ and $r_{s}^{\prime} Q_{I D_{s}}$ by checking whether $\hat{e}\left(r^{\prime}{ }_{s} Q_{I D_{s}}, P\right)=\hat{e}\left(r_{s}{ }_{s} S_{I D_{s}}, P_{p u b}\right)$, one may be tempted to see if $I D_{j}$ is the actual signer by checking whether the following equality holds: $\hat{e}\left(U_{j}+\sum_{i \neq j}\left(U_{i}+h_{i} Q_{I D_{i}}\right), P_{p u b}\right)=\hat{e}(V, P) / \hat{e}\left(h_{j} Q_{I D_{j}}, P_{p u b}\right)$.

However, this method is of no use, as the above equality not only holds when $j=s$, but also $\forall j \in\{1,2, \cdots, n\} \backslash\{s\}$. i.e. the signature is symmetric. Indeed, the above equality is just the same as the equality to be checked in the verification algorithm.

$$
\begin{aligned}
& \hat{e}\left(U_{j}+\sum_{i \neq j}\left(U_{i}+h_{i} Q_{I D_{i}}\right), P_{p u b}\right) \\
= & \hat{e}\left(\sum_{i \neq s} U_{i}+U_{s}+\sum_{i \neq j} h_{i} Q_{I D_{i}}, P_{p u b}\right) \\
= & \hat{e}\left(\sum_{i \neq s} U_{i}+r^{\prime}{ }_{s} Q_{I D_{s}}-\sum_{i \neq s}\left\{U_{i}+h_{i} Q_{I D_{i}}\right\}+\sum_{i \neq j} h_{i} Q_{I D_{i}}, P_{p u b}\right) \\
= & \hat{e}\left(r^{\prime}{ }_{s} Q_{I D_{s}}-\sum_{i \neq s} h_{i} Q_{I D_{i}}+\sum_{i \neq j} h_{i} Q_{I D_{i}}, P_{p u b}\right) \\
= & \hat{e}\left(r^{\prime}{ }_{s} Q_{I D_{s}}+h_{s} Q_{I D_{s}}-h_{j} Q_{I D_{j}}, x P\right) \\
= & \hat{e}\left(r^{\prime}{ }_{s} S_{I D_{s}}+h_{s} S_{I D_{s}}-h_{j} S_{I D_{j}}, P\right) \\
= & \hat{e}\left(V-h_{j} S_{I D_{j}}, P\right)=\hat{e}(V, P) / \hat{e}\left(h_{j} S_{I D_{j}}, P\right)=\hat{e}(V, P) / \hat{e}\left(h_{j} Q_{I D_{j}}, P_{p u b}\right)
\end{aligned}
$$

To conclude, for any fixed message $m$ and fixed set of identities $L$, the distribution of $\left\{\bigcup_{i=1}^{n}\left\{U_{i}\right\}, V\right\}$ are independent and uniformly distributed no matter who is the actual signer. So we conclude that even an adversary with all the private keys corresponding to the set of identities $L$ and unbounded computing resources has no advantage in identifying any one of the participating signers over random guessing. 